\title{
CLASSICAL ASPECTS OF QUANTUM COHOMOLOGY OF GENERALIZED FLAG VARIETIES
}

\author{
NAICHUNG CONAN LEUNG AND CHANGZHENG LI
}

\begin{abstract}
We show that various genus zero Gromov-Witten invariants for flag varieties representing different homology classes are indeed the same. In particular, many of them are classical intersection numbers of Schubert cycles.
\end{abstract}

\section{INTRODUCTION}

A generalized flag variety $G / P$ is the quotient of a simply-connected complex simple Lie group $G$ by a parabolic subgroup $P$ of $G$. The (small) quantum cohomology ring $Q H^{*}(G / P)$ of $G / P$ is a deformation of the ring structure on $H^{*}(G / P)$ by incorporating three-pointed, genus zero Gromov-Witten invariants of $G / P$. The presentation of the ring structure on $Q H^{*}(G / P)$ in special cases have been studied by many mathematicians (see e.g. [35, 24, [10, 5], 9], 33, 28, 17] and references therein). From the viewpoint of enumerative geometry, it is desirable to have (positive) combinatorial formulas for these Gromov-Witten invariants. Thanks to the Peterson-Woodward comparison formula [36, all these Gromov-Witten invariants for $G / P$ can be recovered from the Gromov-Witten invariants for the special case of a complete flag variety $G / B$, where $B$ is a Borel subgroup.

In [30, with the help of the Peterson-Woodward comparison formula, we established a natural filtered algebra structure on $Q H^{*}(G / B)$. In this article, we use the structures of this filtration to obtain relationships among three-pointed genus zero Gromov-Witten invariants $N_{u, v}^{w, \lambda}$ for $G / B$. The Gromov-Witten invariants $N_{u, v}^{w, \lambda}$ are the structure coefficients of the quantum product

$$
\sigma^{u} \star \sigma^{v}=\sum_{\lambda \in H_{2}(G / B, \mathbb{Z}), w} N_{u, v}^{w, \lambda} \mathbf{q}_{\lambda} \sigma^{w}
$$

of the Schubert cocycles $\sigma^{u}$ and $\sigma^{v}$ in the quantum cohomology $Q H^{*}(G / B)$. The evaluation of $\mathbf{q}$ at the origin gives us the classical intersection product

$$
\sigma^{u} \cup \sigma^{v}=\sum_{w} N_{u, v}^{w, 0} \sigma^{w}
$$

Let $\Delta=\left\{\alpha_{1}, \cdots, \alpha_{n}\right\}$ be a base of simple roots of $G$ and $\left\{\alpha_{1}^{\vee}, \cdots, \alpha_{n}^{\vee}\right\}$ be the simple coroots (see section 2.1 and references therein for more details of the notations). The Weyl group $W$ is a Coxeter group generated by simple reflections $\left\{s_{\alpha} \mid \alpha \in \Delta\right\}$. For each $\alpha \in \Delta$, we introduce a map $\operatorname{sgn}_{\alpha}: W \rightarrow\{0,1\}$ defined by $\operatorname{sgn}_{\alpha}(w):=1$ if $\ell(w)-\ell\left(w s_{\alpha}\right)>0$, and 0 otherwise. Here $\ell: W \rightarrow \mathbb{Z}_{>0}$ denotes the length function. Let $\mathfrak{h}$ be the dual of the vector space $\mathfrak{h}^{*}:=\oplus_{\alpha \in \Delta} \mathbb{C} \alpha$ and $\langle\cdot, \cdot\rangle: \mathfrak{h}^{*} \times \mathfrak{h} \rightarrow \mathbb{C}$ be the natural pairing. Note that $H_{2}(G / B, \mathbb{Z})$ can be canonically identified with the coroot lattice $Q^{\vee}:=\oplus_{\alpha \in \Delta} \mathbb{Z} \alpha^{\vee} \subset \mathfrak{h}$. We prove

Key words and phrases. Gromov-Witten invariants. Quantum cohomology. Flag varieties. 
Theorem 1.1. For any $u, v, w \in W$ and for any $\lambda \in Q^{\vee}$, we have

(1) $N_{u, v}^{w, \lambda}=0$ unless $\operatorname{sgn}_{\alpha}(w)+\langle\alpha, \lambda\rangle \leq \operatorname{sgn}_{\alpha}(u)+\operatorname{sgn}_{\alpha}(v)$ for all $\alpha \in \Delta$.

(2) Suppose $\operatorname{sgn}_{\alpha}(w)+\langle\alpha, \lambda\rangle=\operatorname{sgn}_{\alpha}(u)+\operatorname{sgn}_{\alpha}(v)=2$ for some $\alpha \in \Delta$, then

$$
N_{u, v}^{w, \lambda}=N_{u s_{\alpha}, v s_{\alpha}}^{w, \lambda-\alpha^{\vee}}= \begin{cases}N_{u, v s_{\alpha}}^{w s_{\alpha}, \lambda-\alpha^{\vee}}, & \text { if } \operatorname{sgn}_{\alpha}(w)=0 \\ N_{u, v s_{\alpha}}^{w s_{\alpha}, \lambda}, & \text { if } \operatorname{sgn}_{\alpha}(w)=1 .\end{cases}
$$

We obtain nice applications of the above theorem which demonstrate the socalled "quantum to classical" principle.

The "quantum to classical" principle says that certain three-pointed genus zero Gromov-Witten invariants for a given homogeneous space are classical intersection numbers for a typically different homogeneous space. This phenomenon, probably for the first time, occurred in the proof of quantum Pieri rule for partial flag varieties of type $A$ by Ciocan-Fontanine [10, and later occurred in the elementary proof of quantum Pieri rule for complex Grassmannians by Buch [3] as well as the work [26, 27. of Kresch and Tamvakis on Lagrangian and orthogonal Grassmannians. The phrase "quantum to classical principle" was introduced by Chaput and Perrin 9 for the work [4] of Buch, Kresch and Tamvakis on complex Grassmannians, Lagrangian Grassmannians and orthogonal Grassmannians, which says that any three-pointed genus zero Gromov-Witten invariant on a Grassmannian of aforementioned types is equal to a classical intersection number on a partial flag variety of the same Lie type. Recently, this principle has been developed by Buch, Kresch and Tamvakis for isotropic Grassmannians of classical types [5]. For Grassmannians of certain exceptional types, this principle has also been studied by Chaput, Manivel and Perrin (8], 9]). For flag varieties of $A$-type, there are relevant studies by Coskun 13. For the special case of computing the number of lines in a general complete variety $G / B$, this principle has also been studied earlier by the second author and Mihalcea 32. In addition, we note that this principle for certain $K$-theoretic Gromov-Witten invariants have been studied by Buch-Mihalcea ([6], [7]) and by Li-Mihalcea 32].

Using Theorem 1.1, we not only recover most of the above results on the "quantum to classical" principle, but also get new and interesting results. Further applications for general Lie types other than type $A$ will be treated in [31, 15]. For instance in [31, we could see the applications of Theorem 1.1] in seeking quantum Pieri rules with respect to Chern classes of the dual of the tautological subbundles for Grassmannians of classical types, which are not covered in [5] in general.

For type $A_{n}$ case, we note that the Weyl group $W$ is canonically isomorphic to the permutation group $S_{n+1}$ and $G / B=F \ell_{n+1}=\left\{V_{1} \leqslant \cdots \leqslant V_{n} \leqslant \mathbb{C}^{n+1} \mid \operatorname{dim}_{\mathbb{C}} V_{j}=\right.$ $j, j=1, \cdots, n\}$. An element $u \in W=S_{n+1}$ is called a Grassmannian permutation, if there exists $1 \leq k \leq n$ such that $\sigma^{u} \in H^{*}\left(F \ell_{n+1}\right)$ comes from the pull-back $\pi^{*}: H^{*}(G r(k, n+1)) \rightarrow H^{*}\left(F \ell_{n+1}\right)$ induced from the natural projection map $\pi: F \ell_{n+1} \rightarrow\left\{V_{k} \leqslant \mathbb{C}^{n+1} \mid \operatorname{dim} V_{k}=k\right\}=G r(k, n+1)$. Equivalently, a Grassmannian permutation $u \in W$ is an element such that all the reduced expressions $u=s_{i_{1}} s_{i_{2}} \cdots s_{i_{m}}$, where $m=\ell(u)$, end with the same simple reflection $s_{k}$. When written in "one-line" notation, Grassmannian permutations are precisely the permutations with a single descent (see Remark 2.16 for more details). As an application of Theorem 1.1 we have 
Theorem 1.2. Let $u, v, w \in S_{n+1}$ and $\lambda \in Q^{\vee}$. If $u$ is of Grassmannian type, then there exist $v^{\prime}, w^{\prime} \in S_{n+1}$ such that

$$
N_{u, v}^{w, \lambda}=N_{u, v^{\prime}}^{w^{\prime}, 0} .
$$

It is also interesting to investigate the above theorem from the point of view of symmetries on $Q H^{*}\left(F \ell_{n+1}\right)$, analogous with the cyclic symmetries shown by Postnikov 34. In addition, the proof of Theorem 1.2 will also describe how to find $v^{\prime}$ and $w^{\prime}$ (easily). Special cases of Theorem 1.2 enable us to recover the quantum Pieri rule for partial flag varieties of type $A$ as in [10] and the "quantum to classical" principle for complex Grassmannians as in [4.

Geometrically, the Gromov-Witten invariants $N_{u, v}^{w, \lambda}$ count the number of stable holomorphic maps from the projective line $\mathbb{P}^{1}$, or more generally a rational curve, to $G / B$. In particular, they are all non-negative. There have been closed formulas/algorithms on the classical intersection product by Kostant-Kumar 25] and Duan [14] and on the quantum product by the authors [29. Yet sign cancelations are involved in all these formulas/algorithms. For a complete flag variety (of general type), the problem of finding a positive formula on either side remains open. The "quantum to classical" principle could help us to reduce such an issue on the quantum side to the classical side. When $G=S L(n+1, \mathbb{C})$, we note that positive formulas on the classical intersection numbers have been given by Coskun [1], [12].

The proof of Theorem 1.1 uses functorial relationships established by the authors in [30] and it is combinatorial in nature. However, for a special case (of $\lambda=\alpha^{\vee}$ ) of Theorem 1.1, both a geometric proof of it and a combinatorial proof of its equivariant extension can be found in 32 . We also wish to see a geometric proof of this theorem in the future.

\section{ProOFs OF THEOREMS}

In this section, we first fix the notations in section 2.1. Then we prove our first main theorem in section 2.2. Finally in section 2.3, we obtain our second main theorem, as an application of the first main theorem.

2.1. Notations. More details on Lie theory can be found for example in [21, [22].

Let $G$ be a simply-connected complex simple Lie group of rank $n$ and $B \subset$ $G$ be a Borel subgroup. Let $\Delta=\left\{\alpha_{1}, \cdots, \alpha_{n}\right\} \subset \mathfrak{h}^{*}$ be the simple roots and $\left\{\alpha_{1}^{\vee}, \cdots, \alpha_{n}^{\vee}\right\} \subset \mathfrak{h}$ be the simple coroots, where $\mathfrak{h}$ is the corresponding Cartan subalgebra of $(G, B)$. Let $Q^{\vee}=\bigoplus_{i=1}^{n} \mathbb{Z} \alpha_{i}^{\vee}$ and $\rho=\sum_{i=1}^{n} \chi_{i} \in \mathfrak{h}^{*}$. Here $\chi_{i}$ 's are the fundamental weights, which for any $i, j$ satisfy $\left\langle\chi_{i}, \alpha_{j}^{\vee}\right\rangle=\delta_{i, j}$ with respect to the natural pairing $\langle\cdot, \cdot\rangle: \mathfrak{h}^{*} \times \mathfrak{h} \rightarrow \mathbb{C}$. The Weyl group $W$ is generated by $\left\{s_{1}, \cdots, s_{n}\right\}$, where each $s_{i}=s_{\alpha_{i}}$ is a simple reflection on $\mathfrak{h}^{*}$ defined by $s_{i}(\beta)=$ $\beta-\left\langle\beta, \alpha_{i}^{\vee}\right\rangle \alpha_{i}$. The root system is given by $R=W \cdot \Delta=R^{+} \sqcup\left(-R^{+}\right)$, where $R^{+}=R \cap \bigoplus_{i=1}^{n} \mathbb{Z}_{\geq 0} \alpha_{i}$ is the set of positive roots. Each parabolic subgroup $P \supset B$ is in one-to-one correspondence with a subset $\Delta_{P} \subset \Delta$. In fact, $\Delta_{P}$ is the set of simple roots of a Levi subgroup of $P$. Let $\ell: W \rightarrow \mathbb{Z}_{\geq 0}$ be the length function, $W_{P}$ denote the Weyl subgroup generated by $\left\{s_{\alpha} \mid \alpha \in \Delta_{P}\right\}$ and $W^{P}$ denote the subset $\left\{w \in W \mid \ell(w) \leq \ell(v), \forall v \in w W_{P}\right\}$. Each coset in $W / W_{P}$ has a unique minimal length representative in $W^{P}$.

The (co)homology of a (generalized) flag variety $X=G / P$ has an additive basis of Schubert (co)homology classes indexed by $W^{P}: H_{*}(X, \mathbb{Z})=\bigoplus_{v \in W^{P}} \mathbb{Z} \sigma_{v}$, 
$H^{*}(X, \mathbb{Z})=\bigoplus_{u \in W^{P}} \mathbb{Z} \sigma^{u}$ with $\left\langle\sigma^{u}, \sigma_{v}\right\rangle=\delta_{u, v}$ for any $u, v \in W^{P}$ [1]. Note that each $\sigma_{u}$ (resp. $\sigma^{u}$ ) is a class in the $2 \ell(u)^{\text {th }}$-(co)homology. In particular, $H_{2}(X, \mathbb{Z})=\bigoplus_{\alpha_{i} \in \Delta \backslash \Delta_{P}} \mathbb{Z} \sigma_{s_{i}}$ can be canonically identified with $Q^{\vee} / Q_{P}^{\vee}$, where $Q_{P}^{\vee}:=\bigoplus_{\alpha_{i} \in \Delta_{P}} \mathbb{Z} \alpha_{i}^{\vee}$. For each $\alpha_{j} \in \Delta \backslash \Delta_{P}$, we introduce a formal variable $q_{\alpha_{j}^{\vee}+Q_{P}^{\vee}}$. For $\lambda_{P}=\sum_{\alpha_{j} \in \Delta \backslash \Delta_{P}} a_{j} \alpha_{j}^{\vee}+Q_{P}^{\vee} \in H_{2}(X, \mathbb{Z})$, we denote $q_{\lambda_{P}}=\prod_{\alpha_{j} \in \Delta \backslash \Delta_{P}} q_{\alpha_{j}^{\vee}+Q_{P}^{\vee}}^{a_{j}^{j}}$. The (small) quantum cohomology $Q H^{*}(X)=\left(H^{*}(X) \otimes \mathbb{Q}[\mathbf{q}], \star\right)$ of $X$ is a commutative ring and has a $\mathbb{Q}[\mathbf{q}]$-basis of Schubert classes $\sigma^{u}=\sigma^{u} \otimes 1$. The quantum Schubert structure constants $N_{u, v}^{w, \lambda_{P}}$ for the quantum product

$$
\sigma^{u} \star \sigma^{v}=\sum_{w \in W^{P}, \lambda_{P} \in Q^{\vee} / Q_{P}^{\vee}} N_{u, v}^{w, \lambda_{P}} q_{\lambda_{P}} \sigma^{w}
$$

are genus zero Gromov-Witten invariants given by $N_{u, v}^{w, \lambda_{P}}=\int_{\overline{\mathcal{M}}_{0,3}\left(X, \lambda_{P}\right)} \operatorname{ev}_{1}^{*}\left(\sigma^{u}\right) \cup$ $\operatorname{ev}_{2}^{*}\left(\sigma^{v}\right) \cup \operatorname{ev}_{3}^{*}\left(\left(\sigma^{w}\right)^{\sharp}\right)$. Here $\overline{\mathcal{M}}_{0,3}\left(X, \lambda_{P}\right)$ is the moduli space of stable maps of degree $\lambda_{P} \in H_{2}(X, \mathbb{Z})$ of 3 -pointed genus zero curves into $X, \mathrm{ev}_{i}: \overline{\mathcal{M}}_{0,3}\left(X, \lambda_{P}\right) \rightarrow X$ is the $i$-th canonical evaluation map and $\left\{\left(\sigma^{w}\right)^{\sharp} \mid w \in W^{P}\right\}$ are the elements in $H^{*}(X)$ satisfying $\int_{X}\left(\sigma^{w^{\prime}}\right)^{\sharp} \cup \sigma^{w^{\prime \prime}}=\delta_{w^{\prime}, w^{\prime \prime}}$ for any $w^{\prime}, w^{\prime \prime} \in W^{P}$ [18. Note that $N_{u, v}^{w, \lambda_{P}}=0$ unless $q_{\lambda_{P}} \in \mathbb{Q}[\mathbf{q}]$. It is a well-known fact that these Gromov-Witten invariants $N_{u, v}^{w, \lambda_{P}}$ of the flag variety $X$ are enumerative, counting the number of certain holomorphic maps from $\mathbb{P}^{1}$ to $X$. In particular, they are all non-negative integers. (Thus for the special case of a flag variety $X$, we can also define $Q H^{*}(X)$ over $\mathbb{Z}$ whenever we wish.)

In analog with the classical cohomology, there is a natural $\mathbb{Z}$-grading on the quantum cohomolgy $Q H^{*}(X)$, making it a $\mathbb{Z}$-graded ring:

$$
Q H^{*}(X)=\bigoplus_{n \in \mathbb{Z}}\left(\bigoplus_{\operatorname{deg}\left(q_{\lambda_{P}} \sigma^{w}\right)=n} \mathbb{Q} q_{\lambda_{P}} \sigma^{w}\right) .
$$

Here the degree of $q_{\lambda_{P}} \sigma^{w}$, where $\lambda_{P}=\sum_{\alpha_{j} \in \Delta \backslash \Delta_{P}} a_{j} \alpha_{j}^{\vee}+Q_{P}^{\vee} \in H_{2}(X, \mathbb{Z})$, is given by

$$
\operatorname{deg}\left(q_{\lambda_{P}} \sigma^{w}\right)=\ell(w)+\sum_{\alpha_{j} \in \Delta \backslash \Delta_{P}} a_{j}\left\langle\sigma_{s_{j}}, c_{1}(X)\right\rangle,
$$

in which $\langle\cdot, \cdot\rangle$ is the natural pairing between homology and cohomology classes, and an explicit description of the first Chern class $c_{1}(X)$ can be found for example in [19. When $P=B$, we have $\Delta_{P}=\emptyset, Q_{P}^{\vee}=0, W_{P}=\{1\}$ and $W^{P}=W$. In this case, we simply denote $\lambda=\lambda_{P}$ and $q_{j}=q_{\alpha_{j}^{\vee}}$. As a direct consequence of the $\mathbb{Z}$-graded ring structure $(*)$ of $Q H^{*}(G / B)$, for any $u, v, w \in W$ and for any $\lambda \in Q^{\vee}$, we have

$$
N_{u, v}^{w, \lambda}=0 \text { unless } \ell(w)+\langle 2 \rho, \lambda\rangle=\ell(u)+\ell(v) .
$$

2.2. Proof of Theorem 1.1. This subsection is devoted to the proof of Theorem 1.1. The main arguments are given in section 2.2.3 based on the results in [30] which will be reviewed in section 2.2.2. We will introduce the Peterson-Woodward comparison formula first in section 2.2.1. This comparison formula not only plays an important role in obtaining the results in [30, but also shows us that it suffices to know all quantum Schubert structure constants $N_{u, v}^{w, \lambda}$ for $G / B$ in order to know all quantum Schubert structure constants for all $G / P$ 's. 
2.2.1. Peterson-Woodward comparison formula. We use $\star_{P}$ to distinguish the quantum products for different flag varieties $G / P^{\prime}$ 's (when needed). For any $u, v \in W^{P}$, we have $\sigma^{u} \star_{P} \sigma^{v}=\sum_{w \in W^{P}, \lambda_{P} \in Q^{\vee} / Q_{P}^{\vee}} N_{u, v}^{w, \lambda_{P}} q_{\lambda_{P}} \sigma^{w}$. Note $W^{P} \subset W$. The classes $\sigma^{u}$ and $\sigma^{v}$ in $Q H^{*}(G / P)$ can both be treated as classes in $Q H^{*}(G / B)$ naturally. Whenever referring to $N_{u, v}^{w, \lambda}$ where $\lambda \in Q^{\vee}$, we are considering the quantum product in $Q H^{*}(G / B): \sigma^{u} \star_{B} \sigma^{v}=\sum_{w \in W, \lambda \in Q^{\vee}} N_{u, v}^{w, \lambda} q_{\lambda} \sigma^{w}$.

Proposition 2.1 (Peterson-Woodward comparison formula [36]; see also [28]).

(1) Let $\lambda_{P} \in Q^{\vee} / Q_{P}^{\vee}$. Then there is a unique $\lambda_{B} \in Q^{\vee}$ such that $\lambda_{P}=\lambda_{B}+Q_{P}^{\vee}$ and $\left\langle\gamma, \lambda_{B}\right\rangle \in\{0,-1\}$ for all $\gamma \in R_{P}^{+}\left(=R^{+} \cap \bigoplus_{\beta \in \Delta_{P}} \mathbb{Z} \beta\right)$.

(2) For every $u, v, w \in W^{P}$, we have

$$
N_{u, v}^{w, \lambda_{P}}=N_{u, v}^{w \omega_{P} \omega_{P^{\prime}}, \lambda_{B}} .
$$

Here $\omega_{P}$ (resp. $\omega_{P^{\prime}}$ ) is the longest element in the Weyl subgroup $W_{P}$ (resp. $\left.W_{P^{\prime}}\right)$, where $\Delta_{P^{\prime}}=\left\{\beta \in \Delta_{P} \mid\left\langle\beta, \lambda_{B}\right\rangle=0\right\}$.

Thanks to the above proposition, we obtain an injection of vector spaces

$$
\psi_{\Delta, \Delta_{P}}: Q H^{*}(G / P) \longrightarrow Q H^{*}(G / B) \text { defined by } q_{\lambda_{P}} \sigma^{w} \mapsto q_{\lambda_{B}} \sigma^{w \omega_{P} \omega_{P^{\prime}}} .
$$

For the special case of a singleton subset $\{\alpha\} \subset \Delta$, we denote $P_{\alpha}=P$ and simply denote $\psi_{\alpha}=\psi_{\Delta,\{\alpha\}}$. In this case, we note that $R_{P_{\alpha}}^{+}=\{\alpha\}, Q_{P_{\alpha}}^{\vee}=\mathbb{Z} \alpha^{\vee}$, and we have the natural fibration $P_{\alpha} / B \rightarrow G / B \rightarrow G / P_{\alpha}$ with $P_{\alpha} / B \cong \mathbb{P}^{1}$.

Example 2.2. Let $\lambda_{P_{\alpha}}=\beta^{\vee}+Q_{P_{\alpha}}^{\vee}$ where $\beta \in \Delta \backslash\{\alpha\}$. Then we have $\left\langle\alpha, \beta^{\vee}\right\rangle \in$ $\{0,-1,-2,-3\}$. Furthermore, we have

$$
\psi_{\alpha}\left(q_{\lambda_{P_{\alpha}}}\right)= \begin{cases}q_{\beta^{\vee}}, & \text { if }\left\langle\alpha, \beta^{\vee}\right\rangle=0 \\ s_{\alpha} q_{\beta^{\vee}}, & \text { if }\left\langle\alpha, \beta^{\vee}\right\rangle=-1 \\ q_{\alpha^{\vee}} q_{\beta^{\vee}}, & \text { if }\left\langle\alpha, \beta^{\vee}\right\rangle=-2 \\ s_{\alpha} q_{\alpha} q_{\beta^{\vee}}, & \text { if }\left\langle\alpha, \beta^{\vee}\right\rangle=-3 .\end{cases}
$$

More generally, we consider $\lambda_{P_{\alpha}}=\lambda^{\prime}+Q_{P_{\alpha}}^{\vee} \in Q^{\vee} / Q_{P_{\alpha}}^{\vee}$, where $\lambda^{\prime}=\sum_{\beta \in \Delta \backslash\{\alpha\}} c_{\beta} \beta^{\vee} \in$ $Q^{\vee}$. Setting $m=\left\langle\alpha, \lambda^{\prime}\right\rangle$, we have $\psi_{\alpha}\left(q_{\lambda_{P_{\alpha}}} \sigma^{w}\right)=\left\{\begin{array}{ll}q_{\lambda^{\prime}-\frac{m}{2}} \alpha^{\vee} \sigma^{w}, & \text { if } m \text { is even } \\ q_{\lambda^{\prime}-\frac{m+1}{2} \alpha^{\vee}} \sigma^{w s_{\alpha}}, & \text { if } m \text { is odd }\end{array}\right.$.

2.2.2. $\mathbb{Z}^{2}$-filtrations on $Q H^{*}(G / B)$. As shown in 30, given any parabolic subgroup $P$ of $G$ containing $B$, we can construct a $\mathbb{Z}^{\left|\Delta_{P}\right|+1}$-filtration on $Q H^{*}(G / B)$. In this subsection, we review the main results in [30] for the special case of a parabolic subgroup that corresponds to a singleton subset $\{\alpha\}$. Using them, we prove Theorem 1.1 in next subsection.

Recall that a natural basis of $Q H^{*}(G / B)\left[q_{1}^{-1}, \cdots, q_{n}^{-1}\right]$ is given by $q_{\lambda} \sigma^{w}$ 's labelled by $(w, \lambda) \in W \times Q^{\vee}$. Note that $q_{\lambda} \sigma^{w} \in Q H^{*}(G / B)$ if and only if $q_{\lambda} \in \mathbb{Q}[\mathbf{q}]$ is a polynomial. In order to obtain a filtration on $Q H^{*}(G / B)$, we just need to define (nice) gradings for a given basis of it. Furthermore as in the introduction, we have defined a map $\operatorname{sgn}_{\alpha}$ with respect to any given simple root $\alpha \in \Delta$ as follows.

$$
\operatorname{sgn}_{\alpha}: W \rightarrow\{0,1\} ; \operatorname{sgn}_{\alpha}(w)=\left\{\begin{array}{ll}
1, & \text { if } \ell(w)-\ell\left(w s_{\alpha}\right)>0 \\
0, & \text { if } \ell(w)-\ell\left(w s_{\alpha}\right) \leq 0
\end{array} .\right.
$$


Note that $\ell(w)-\ell\left(w s_{\alpha}\right)= \pm 1$ and that $\ell(w)-\ell\left(w s_{\alpha}\right)=1$ if and only if $w(\alpha) \in-R^{+}$, which holds if and only if $w=u s_{\alpha}$ for a unique $u \in W^{P_{\alpha}}$. (See e.g. 23.) We can define a grading map $g r_{\alpha}$ with respect to a given simple root $\alpha \in \Delta$ as follows.

$$
\begin{aligned}
g r_{\alpha}: W \times Q^{\vee} \longrightarrow \mathbb{Z}^{2} ; \\
g r_{\alpha}\left(q_{\lambda} \sigma^{w}\right)=\left(\operatorname{sgn}_{\alpha}(w)+\langle\alpha, \lambda\rangle, \ell(w)+\langle 2 \rho, \lambda\rangle-\operatorname{sgn}_{\alpha}(w)-\langle\alpha, \lambda\rangle\right) .
\end{aligned}
$$

Here we are using lexicographical order on $\mathbb{Z}^{2}$. That is, $\mathbf{a}=\left(a_{1}, a_{2}\right)<\mathbf{b}=\left(b_{1}, b_{2}\right)$ if and only if either $\left(a_{1}=b_{1}\right.$ and $\left.a_{2}<b_{2}\right)$ or $a_{1}<b_{1}$ holds. The above $\mathbb{Z}^{2}$-grading of $q_{\lambda} \sigma^{w}$ can recover the degree grading of it as in section 2.1. Precisely, if we write $g r_{\alpha}\left(q_{\lambda} \sigma^{w}\right)=(i, j)$, then $\operatorname{deg}\left(q_{\lambda} \sigma^{w}\right)=i+j$.

Remark 2.3. Following from Corollary 3.13 of [30], our grading map gr $r_{\alpha}$ coincides with the grading map in Definition 2.8 of [30] by using the Peterson-Woodward lifting map $\psi_{\alpha}=\psi_{\Delta,\{\alpha\}}$.

As a consequence, we obtain a family $\mathcal{F}=\left\{F_{\mathbf{a}}\right\}_{\mathbf{a} \in \mathbb{Z}^{2}}$ of vector subspaces of $Q H^{*}(G / B)$, where $F_{\mathbf{a}}:=\underset{g r_{\alpha}\left(q_{\lambda} \sigma^{w}\right) \leq \mathbf{a}}{\bigoplus} \mathbb{Q} q_{\lambda} \sigma^{w} \subset Q H^{*}(G / B)$, and the associated graded vector space $G r^{\mathcal{F}}\left(Q H^{*}(G / B)\right)=\bigoplus_{\mathbf{a} \in \mathbb{Z}^{2}} G r_{\mathbf{a}}^{\mathcal{F}}$ with respect to $\mathcal{F}$, where $G r_{\mathbf{a}}^{\mathcal{F}}:=F_{\mathbf{a}} / \cup_{\mathbf{b}<\mathbf{a}} F_{\mathbf{b}}$

Proposition 2.4 (Theorem 1.2 of [30]). $Q H^{*}(G / B)$ is a $\mathbb{Z}^{2}$-filtered algebra with respect to $\mathcal{F}$. That is, we have $F_{\mathbf{a}} \star F_{\mathbf{b}} \subset F_{\mathbf{a}+\mathbf{b}}$ for any $\mathbf{a}, \mathbf{b} \in \mathbb{Z}^{2}$.

$$
\text { Denote } G r_{\text {vert }}^{\mathcal{F}}\left(Q H^{*}(G / B)\right):=\bigoplus_{i \in \mathbb{Z}} G r_{(i, 0)}^{\mathcal{F}} \text { and } G r_{\text {hor }}^{\mathcal{F}}\left(Q H^{*}(G / B)\right):=\bigoplus_{j \in \mathbb{Z}} G r_{(0, j)}^{\mathcal{F}} \text {. }
$$

We take the canonical isomorphism $Q H^{*}\left(\mathbb{P}^{1}\right) \cong \frac{\mathbb{Q}[x, t]}{\left\langle x^{2}-t\right\rangle}$ for the fiber of the fibration $\mathbb{P}^{1} \rightarrow G / B \rightarrow G / P_{\alpha}$.

Proposition 2.5 (Theorem 1.4 of [30]). The following maps $\Psi_{\mathrm{vert}}^{\alpha}$ and $\Psi_{\mathrm{hor}}^{\alpha}$ are well-defined and they are algebra isomorphism $₫$.

$$
\begin{array}{ccc}
\Psi_{\text {vert }}^{\alpha}: & Q H^{*}\left(\mathbb{P}^{1}\right) \longrightarrow G r_{\text {vert }}^{\mathcal{F}}\left(Q H^{*}(G / B)\right) ; & x \mapsto \overline{s_{\alpha}}, t \mapsto \overline{q_{\alpha}} . \\
\Psi_{\text {hor }}^{\alpha}: & Q H^{*}\left(G / P_{\alpha}\right) \longrightarrow G r_{\text {hor }}^{\mathcal{F}}\left(Q H^{*}(G / B)\right) ; & q_{\lambda_{P_{\alpha}}} \sigma^{w} \mapsto \overline{\psi_{\alpha}\left(q_{\lambda_{P_{\alpha}}} \sigma^{w}\right)} .
\end{array}
$$

Here we note that $\overline{s_{\alpha}} \in G r_{(1,0)}^{\mathcal{F}} \subset G r_{\text {vert }}^{\mathcal{F}}\left(Q H^{*}(G / B)\right)$ denotes the graded component of $\sigma^{s_{\alpha}}+\cup_{\mathbf{b}<(1,0)} F_{\mathbf{b}}$. Similar notations are taken whenever " $\overline{()}$ " is used. In addition, we have

Proposition 2.6 (Proposition 3.23 of [30]). For any $u \in W^{P_{\alpha}}$, we have $\sigma^{u} \star \sigma^{s_{\alpha}}=$ $\sigma^{u s_{\alpha}}+\sum_{w, \lambda} b_{w, \lambda} q_{\lambda} \sigma^{w}$ with $g r_{\alpha}\left(q_{\lambda} \sigma^{w}\right)<g r_{\alpha}\left(\sigma^{u s_{\alpha}}\right)$ whenever $b_{w, \lambda} \neq 0$.

The next lemma follows directly from the definition of the grading map $g r_{\alpha}$.

Lemma 2.7. Let $u, v, w \in W$ and $\lambda \in Q^{\vee}$. Then $g r_{\alpha}\left(\sigma^{u}\right)+g r_{\alpha}\left(\sigma^{v}\right)=g r_{\alpha}\left(q_{\lambda} \sigma^{w}\right)$ if and only if both $\ell(w)+\langle 2 \rho, \lambda\rangle=\ell(u)+\ell(v)$ and $\operatorname{sgn}_{\alpha}(w)+\langle\alpha, \lambda\rangle=\operatorname{sgn}_{\alpha}(u)+\operatorname{sgn}_{\alpha}(v)$ hold.

\footnotetext{
${ }^{1}$ In terms of notations in [30], $\Psi_{\text {vert }}^{\alpha}=\Psi_{1}$ and $\Psi_{\text {hor }}^{\alpha}=\Psi_{2}$.
} 
2.2.3. Proof of Theorem 1.1. The first half of Theorem 1.1 is a direct consequence of Proposition 2.4. Indeed, if $\operatorname{sgn}_{\beta}(w)+\langle\beta, \lambda\rangle>\operatorname{sgn}_{\beta}(u)+\operatorname{sgn}_{\beta}(v)$ for some $\beta \in$ $\Delta$, then $g r_{\beta}\left(q_{\lambda} \sigma^{w}\right)>g r_{\beta}\left(\sigma^{u}\right)+g r_{\beta}\left(\sigma^{v}\right)$. Since $\sigma^{u} \star \sigma^{v} \in F_{g r_{\beta}\left(\sigma^{u}\right) \star F_{g r_{\beta}\left(\sigma^{v}\right)} \subset}$ $F_{g r_{\beta}\left(\sigma^{u}\right)+g r_{\beta}\left(\sigma^{v}\right)}$, we conclude $N_{u, v}^{w, \lambda}=0$.

It remains to show the second half of Theorem 1.1. Note that $\operatorname{sgn}_{\alpha}$ is a map from $W$ to $\{0,1\}$. Since $\operatorname{sgn}_{\alpha}(u)+\operatorname{sgn}_{\alpha}(v)=2$, we have $\operatorname{sgn}_{\alpha}(u)=\operatorname{sgn}_{\alpha}(v)=1$. Consequently, $u^{\prime}:=u s_{\alpha}$ and $v^{\prime}:=v s_{\alpha}$ are both elements in $W^{P_{\alpha}}$. In the rest, we can assume $\ell(w)+\langle 2 \rho, \lambda\rangle=\ell(u)+\ell(v)$. (Otherwise, both $N_{u, v}^{w, \lambda}$ and $N_{u^{\prime}, v^{\prime}}^{w, \lambda-\alpha^{v}}$ would vanish, directly following from the standard $\mathbb{Z}$-graded ring structure $(*)$ of $Q H^{*}(G / B)$.)

By Proposition 2.6, we have

$$
\overline{\sigma^{u^{\prime}} \star \sigma^{s_{\alpha}}}=\overline{\sigma^{u}} \in G r_{g r_{\alpha}\left(\sigma^{u}\right)}^{\mathcal{F}} \text { and } \overline{\sigma^{v^{\prime} \star \sigma^{s_{\alpha}}}}=\overline{\sigma^{v}} \in G r_{g r_{\alpha}\left(\sigma^{v}\right)}^{\mathcal{F}} .
$$

Note that $Q H^{*}(G / B)$ is an associative and commutative $\mathbb{Z}^{2}$-filtered algebra with respect to $\mathcal{F}$. As a consequence, $G r^{\mathcal{F}}\left(Q H^{*}(G / B)\right)$ is an associative and commutative $\mathbb{Z}^{2}$-graded algebra. Thus we have

$$
\text { LHS }:=\overline{\sigma^{u^{\prime}} \star \sigma^{s_{\alpha}}} \star \overline{\sigma^{v^{\prime}} \star \sigma^{s_{\alpha}}}=\overline{\sigma^{u}} \star \overline{\sigma^{v}}=: \text { RHS }
$$

in $G r_{g r_{\alpha}\left(\sigma^{u}\right)+g r_{\alpha}\left(\sigma^{v}\right)}^{\mathcal{F}}$. By Lemma 2.7, we have

$$
\mathrm{RHS}=\overline{\sigma^{u} \star \sigma^{v}}=\overline{\sum N_{u, v}^{\tilde{w}, \tilde{\lambda}} q_{\tilde{\lambda}} \sigma^{\tilde{w}}}=\sum N_{u, v}^{\tilde{w}, \tilde{\lambda}} \overline{q_{\tilde{\lambda}} \sigma^{\tilde{w}}},
$$

where the summation is over those $(\tilde{w}, \tilde{\lambda}) \in W \times Q^{\vee}$ satisfying $\ell(\tilde{w})+\langle 2 \rho, \tilde{\lambda}\rangle=$ $\ell(u)+\ell(v)$ and $\operatorname{sgn}_{\alpha}(\tilde{w})+\langle\alpha, \tilde{\lambda}\rangle=2$. Let $\star_{\alpha}$ denote the quantum product for $Q H^{*}\left(G / P_{\alpha}\right)$. By Proposition 2.5, we have

$$
\text { LHS }=\left(\overline{\sigma^{u^{\prime}}} \star \overline{\sigma^{v^{\prime}}}\right) \star\left(\overline{\sigma^{s_{\alpha}} \star \overline{\sigma^{s_{\alpha}}}}\right)=\Psi_{\text {hor }}^{\alpha}\left(\sigma^{u^{\prime}} \star_{\alpha} \sigma^{v^{\prime}}\right) \star \overline{q_{\alpha^{v}}}=\sum N_{u^{\prime}, v^{\prime}}^{w^{\prime}, \lambda_{P_{\alpha}}} \overline{\psi_{\alpha}\left(\sigma^{w^{\prime}} q_{\lambda_{P_{\alpha}}}\right) q_{\alpha^{\vee}}},
$$

the summation over those $\left(w^{\prime}, \lambda_{P_{\alpha}}\right) \in W^{P} \times Q^{\vee} / Q_{P_{\alpha}}^{\vee}$ (with $\lambda_{P_{\alpha}}$ being effective). Then we conclude $N_{u, v}^{w, \lambda}=N_{u s_{\alpha}, v s_{\alpha}}^{w, \lambda-\alpha^{\vee}}$ by comparing coefficients of both sides. Indeed, for $\lambda_{P_{\alpha}}:=\lambda+Q_{P_{\alpha}}^{\vee}$, we have $\lambda_{B}=\lambda-\alpha^{\vee}$ via Peterson-Woodward comparison formula (by noting $\left\langle\alpha, \lambda-\alpha^{\vee}\right\rangle=-\operatorname{sgn}_{\alpha}(w) \in\{0,-1\}$ ). Set $w^{\prime}:=w$ if $\operatorname{sgn}_{\alpha}(w)=0$, or $w s_{\alpha}$ if $\operatorname{sgn}_{\alpha}(w)=1$. Note that $\psi_{\alpha}\left(\sigma^{w^{\prime}} q_{\lambda_{P_{\alpha}}}\right) q_{\alpha^{\vee}}=\sigma^{w} q_{\lambda}$. We conclude

$$
N_{u, v}^{w, \lambda}=N_{u^{\prime}, v^{\prime}}^{w^{\prime}, \lambda_{P_{\alpha}}}=N_{u^{\prime}, v^{\prime}}^{w, \lambda} \text {. }
$$

Note that for any $\hat{w} \in W$, we have



Hence, we have

$$
\begin{aligned}
\overline{\sigma^{u}} \star \overline{\sigma^{v}}=\overline{\sigma^{u}} \star \overline{\sigma^{v^{\prime}} \star \sigma^{s_{\alpha}}} & =\overline{\sigma^{u} \star \sigma^{v^{\prime}} \star \overline{\sigma^{s_{\alpha}}}} \\
& =\overline{\sum N_{u, v^{\prime}}^{\hat{w}, \hat{\lambda}} q_{\hat{\lambda}} \sigma^{\hat{w}} \star \overline{\sigma^{s_{\alpha}}}} \\
& =\sum N_{u, v^{\prime}}^{\hat{w}, \hat{\lambda}} \overline{q_{\hat{\lambda}} \sigma^{\hat{w} s_{\alpha}}}+\sum N_{u, v^{\prime}}^{\hat{w}, \hat{\lambda}} \overline{q_{\hat{\lambda}+\alpha^{\vee}} \sigma^{\hat{w} s_{\alpha}}},
\end{aligned}
$$

the former (resp. latter) summation over those $(\hat{w}, \hat{\lambda}) \in W \times Q^{\vee}$ satisfying $\ell(\hat{w})+$ $\langle 2 \rho, \hat{\lambda}\rangle=\ell(u)+\ell\left(v^{\prime}\right), \operatorname{sgn}_{\alpha}(\hat{w})+\langle\alpha, \hat{\lambda}\rangle=1$ and $\operatorname{sgn}_{\alpha}(\hat{w})=0$ (resp. 1). Hence, if $\operatorname{sgn}_{\alpha}(w)=0$ (resp. 1), then we have $N_{u, v}^{w, \lambda} q_{\lambda} \sigma^{w}=N_{u, v^{\prime}}^{\hat{w}, \hat{\lambda}} q_{\hat{\lambda}+\alpha^{\vee}} \sigma^{\hat{w} s_{\alpha}}$ (resp. 
$\left.N_{u, v^{\prime}}^{\hat{w}, \hat{\lambda}} q_{\hat{\lambda}} \sigma^{\hat{w} s_{\alpha}}\right)$ for a unique $(\hat{w}, \hat{\lambda})$ in the latter (resp. former) summation. Thus we conclude that $N_{u, v}^{w, \lambda}$ equals $N_{u, v s_{\alpha}}^{w s_{\alpha}, \lambda-\alpha^{\vee}}$ if $\operatorname{sgn}_{\alpha}(w)=0$, or $N_{u, v s_{\alpha}}^{w s_{\alpha}, \lambda}$ if $\operatorname{sgn}_{\alpha}(w)=1$.

2.3. Applications. In this subsection, we give applications of Theorem 1.1 for $\Delta$ of $A$-type case. (See the introduction for possible further applications for other cases.)

For convenience, we assume the Dynkin diagram of $\Delta$ is given by $\begin{array}{ccc}\begin{array}{c}0 \\ \alpha_{1}\end{array} \alpha_{2} & \cdots \circ \\ \longrightarrow & \alpha_{n}\end{array}$.

The flag variety of $A_{n}$-type, corresponding to a subset $\Delta \backslash\left\{\alpha_{a_{1}}, \cdots, \alpha_{a_{k}}\right\}$, parameterizes flag of linear subspaces $\left\{V_{a_{1}} \leqslant \cdots \leqslant V_{a_{r}} \leqslant \mathbb{C}^{n+1} \mid \operatorname{dim}_{\mathbb{C}} V_{a_{j}}=a_{j}, j=\right.$ $1, \cdots, r\}$ where $\left[a_{1}, \cdots, a_{r}\right]$ is a subsequence of $[1, \cdots, n]$. We fix an $\alpha_{k}$ once and for all. Let $P \supset B$ denote the parabolic subgroup that corresponds to $\Delta_{P}=\Delta \backslash\left\{\alpha_{k}\right\}$. Note that the complete flag variety $F \ell_{n+1}=G / B$ and the complex Grassmannian $G r(k, n+1)=G / P$ correspond to the subsequences $[1,2, \cdots, n]$ and $[k]$ respectively, where $G=S L(n+1, \mathbb{C})$. The natural projection $\pi: G / B \rightarrow G / P$ is just the forgetting map, sending a flag $V_{1} \leqslant \cdots \leqslant V_{n} \leqslant \mathbb{C}^{n+1}$ in $F \ell_{n+1}$ to the point $V_{k} \leqslant \mathbb{C}^{n+1}$ in $\operatorname{Gr}(k, n+1)$. Furthermore, the induced map $\pi^{*}: H^{*}(G / P) \rightarrow H^{*}(G / B)$ sends a Schubert class $\sigma_{P}^{w} \in H^{*}(G / P)$ (where $w \in W^{P}$ ) to the Schubert class $\pi^{*}\left(\sigma_{P}^{w}\right)=\sigma_{B}^{w} \in H^{*}(G / B)$. Such a class $\sigma^{w}$ in $H^{*}(G / B)$ (with $w \in W^{P}$ ) is called a Grassmannian class. By abuse of notations, we skip the subscript " $B$ " and " $P$ ". Using Theorem 1.1, we can show Theorem 1.2 stated in the introduction, a reformulation of which is given as follows.

Theorem 1.2. For any $u \in W^{P}, v, w \in W$ and $\lambda \in Q^{\vee}$, there exist $v^{\prime}, w^{\prime} \in W$ such that

$$
N_{u, v}^{w, \lambda}=N_{u, v^{\prime}}^{w^{\prime}, 0} .
$$

To prove the above theorem, we need the following two lemmas.

Lemma 2.8. Given any nonzero $\lambda=\sum_{j=1}^{n} a_{j} \alpha_{j}^{\vee} \in Q^{\vee}$ with $a_{j} \geq 0$ for all $j$, there exists $m \in\{1, \cdots, n\}$ such that $\left\langle\alpha_{m}, \lambda\right\rangle>0$ and $a_{m}>0$.

Proof. Assume $\left\langle\alpha_{m}, \lambda\right\rangle \leq 0$ for all $m$. Then $\lambda$ is a non-positive sum of fundamental coweights. As a consequence, $\lambda$ is a non-positive sum of simple coroots $\alpha_{j}^{\vee}$ 's, by Table 1 in section 13.2 of 21. Thus $\lambda=0$, which contradicts the assumptions.

Hence, there exists $m$ such that $\left\langle\alpha_{m}, \lambda\right\rangle>0$. Consequently, we have $a_{m}>0$, by noting that $\left\langle\alpha_{m}, \alpha_{j}^{\vee}\right\rangle$ is positive if $j=m$, or non-positive otherwise.

Remark 2.9. Lemma 2.8 works for $\Delta$ of all types with the same proof.

Lemma 2.10. Let $\lambda=\sum_{j=1}^{n} a_{j} \alpha_{j}^{\vee} \in Q^{\vee}$ with $a_{j} \geq 0$ for all $j$. If $\left\langle\alpha_{m}, \lambda\right\rangle>0$ for a unique $m$, then we have $\left\langle\alpha_{m}, \lambda\right\rangle \geq 2$.

Proof. Let $\operatorname{Dyn}(\tilde{\Delta})$ denote the Dynkin diagram associated to a subbase $\tilde{\Delta} \subset \Delta$.

Denote $\Delta^{\prime}:=\left\{\alpha_{j} \mid a_{j}>0\right\}$. Clearly, $\alpha_{m} \in \Delta^{\prime}$. We first conclude that $\operatorname{Dyn}\left(\Delta^{\prime}\right)$ is connected. (Otherwise, we can write $\Delta^{\prime}=\Delta_{1} \sqcup \Delta_{2}$ with $\operatorname{Dyn}\left(\Delta_{1}\right)$ being a connected component of $\operatorname{Dyn}\left(\Delta^{\prime}\right)$. Then $\lambda=\lambda_{1}+\lambda_{2}$ with $\lambda_{1}$ (resp. $\lambda_{2}$ ) belonging to the coroot sub-lattice of $\Delta_{1}$ (resp. $\Delta_{2}$ ). Note that $\Delta_{1}$ and $\Delta_{2}$ are orthogonal to each other. For each $j \in\{1,2\}$, there exists $\alpha_{m_{j}} \in \Delta_{j}$ such that $\left\langle\alpha_{m_{j}}, \lambda_{j}\right\rangle>0$ by Lemma 2.8. This contradicts the uniqueness of $\alpha_{m}$.) Thus $\Delta^{\prime}=\left\{\alpha_{i}, \alpha_{i+1}, \cdots, \alpha_{p}\right\}$ for some $1 \leq i \leq m \leq p \leq n$.

When $i=p$, the statements holds, by noting that $\left\langle\alpha_{m}, \alpha_{m}^{\vee}\right\rangle=2$ and $\lambda=a_{m} \alpha_{m}^{\vee}$ in this case. When $i<p$, we can assume $i<m$ without loss of generality. Since 
$0 \geq\left\langle\alpha_{i}, \lambda\right\rangle=2 a_{i}-a_{i+1}$, we have $a_{i+1} \geq 2 a_{i}>a_{i}>0$. Since $0 \geq\left\langle\alpha_{i+1}, \lambda\right\rangle=$ $-a_{i}+2 a_{i+1}-a_{i+2}$, we have $a_{i+2} \geq a_{i+1}+\left(a_{i+1}-a_{i}\right)>a_{i+1}>0$. By induction, we conclude $a_{m}>a_{m-1}>0$. If $m=p$, then we have $\left\langle\alpha_{m}, \lambda\right\rangle=2 a_{m}-a_{m-1} \geq$ $2\left(a_{m-1}+1\right)-a_{m-1}>2$. If $m<p$, then we can show $a_{m}>a_{m+1}$ with the same arguments. As a consequence, we have $\left\langle\alpha_{m}, \lambda\right\rangle=-a_{m-1}+2 a_{m}-a_{m+1} \geq$ $-a_{m-1}+\left(a_{m-1}+1+a_{m+1}+1\right)-a_{m+1} \geq 2$.

Proof of Theorem 1.2. Clearly, the statement holds if $N_{u, v}^{w, \lambda}$ vanishes or $\lambda=0$.

Given nonzero $\lambda=\sum_{j=1}^{n} a_{j} \alpha_{j}^{\vee} \in Q^{\vee}$, we can assume $a_{j} \geq 0$ for all $j$, i.e. $\lambda$ is effective, because otherwise $N_{u, v}^{w, \lambda}$ vanishes. Since $\lambda \neq 0$, there exists $m$ such that $\left\langle\alpha_{m}, \lambda\right\rangle>0$ by Lemma 2.8. We simply denote $\operatorname{sgn}_{m}:=\operatorname{sgn}_{\alpha_{m}}$, which is a map from $W$ to $\{0,1\}$ defined in the introduction (see also section 2.2.2).

If such an $m$ is not unique, then we can take any one such $m$ that is not equal to $k$. Since $u \in W^{P}$ where $\Delta_{P}=\Delta \backslash\left\{\alpha_{k}\right\}$, we have $\operatorname{sgn}_{m}(u)=0$. If $\operatorname{sgn}_{m}(v)<$ $\operatorname{sgn}_{m}(w)+\left\langle\alpha_{m}, \lambda\right\rangle$, then we have $N_{u, v}^{w, \lambda}=0$ by Theorem 1.1 (1); and hence we are done. Otherwise, we have $\operatorname{sgn}_{m}(v)=\operatorname{sgn}_{m}(w)+\left\langle\alpha_{m}, \lambda\right\rangle=1$ and $\operatorname{sgn}_{m}(w)=0$. By Theorem 1.1 (2), we have $N_{u s_{m}, v}^{w s_{m}, \lambda}=N_{u, v s_{m}}^{w s_{m}, \lambda-\alpha_{m}^{\vee}}=N_{u, v}^{w, \lambda}$.

If such an $m$ is unique, then we have $\left\langle\alpha_{m}, \lambda\right\rangle \geq 2$ by Lemma 2.10. Thus either $\operatorname{sgn}_{m}(u)+\operatorname{sgn}_{m}(v)<\operatorname{sgn}_{m}(w)+\left\langle\alpha_{m}, \lambda\right\rangle$ or $\operatorname{sgn}_{m}(u)+\operatorname{sgn}_{m}(v)=\operatorname{sgn}_{m}(w)+\left\langle\alpha_{m}, \lambda\right\rangle$ holds. For the former case, $N_{u, v}^{w, \lambda}$ vanishes and then it is done. For the latter case, we conclude $m=k, \operatorname{sgn}_{k}(v)=1, \operatorname{sgn}_{k}(w)=0$ and $\left\langle\alpha_{k}, \lambda\right\rangle=2$, by noting that $\operatorname{sgn}_{j}(u)=1$ if $j=k$, or 0 otherwise. Thus we have $N_{u, v}^{w, \lambda}=N_{u s_{k}, v s_{k}}^{w, \lambda-\alpha_{k}^{\vee}}=N_{u, v s_{k}}^{w s_{k}, \lambda-\alpha_{k}^{\vee}}$, by using Theorem 1.1 (2) again.

Hence, either of the followings must hold: (i) $N_{u, v}^{w, \lambda}=0$ (and then it is done); (ii) $N_{u, v}^{w, \lambda}=N_{u, v s_{m}}^{w s_{m}, \lambda^{\prime}}$ with $\lambda^{\prime}=\lambda-\alpha_{m}^{\vee}=\sum_{j} a_{j}^{\prime} \alpha_{j}^{\vee}$, in which $\left|\lambda^{\prime}\right|=|\lambda|-1$ with $a_{j}^{\prime}=a_{j}-1 \geq 0$ if $j=m$, or $a_{j}$ otherwise. Here $|\lambda|:=\sum_{j=1}^{n} a_{j}$. Therefore, the statement holds, by using induction on $|\lambda|$.

Besides Theorem 1.2, we can also find other applications of Theorem 1.1

Example 2.11. Let $G / B=F \ell_{4}$. Take $u=v=s_{2} s_{1} s_{2}, w=s_{2} s_{3}$ and $\lambda=\alpha_{1}^{\vee}+\alpha_{2}^{\vee}$. (Note that neither of the Schubert classes $\sigma^{u}, \sigma^{v}$ are Grassmannian classes.) We have

$$
N_{u, v}^{w, \lambda}=N_{u, v s_{3}}^{w s_{3}, \lambda+\alpha_{3}^{\vee}}=N_{s_{2} s_{1} s_{2}, s_{2} s_{1} s_{2} s_{3}}^{s_{2}, \alpha^{\vee}},
$$

in which we increase the degree $q_{\lambda}$ first. Then we have

$$
N_{u, v}^{w, \lambda}=N_{s_{2} s_{1}, s_{2} s_{1} s_{2} s_{3}}^{1, \alpha_{3}^{\vee}}=N_{s_{2} s_{1}, s_{2} s_{1} s_{2}}^{s_{3}, \alpha_{2}^{\vee}+\alpha_{2}^{\vee}}=N_{s_{2} s_{1}, s_{2} s_{1}}^{s_{3} s_{2}, \alpha_{1}^{\vee}}=N_{s_{2}, s_{2}}^{s_{3} s_{2}, 0}=1 .
$$

In fact, we already know all the nonzero three-pointed, genus zero Gromov-Witten invariants for $F \ell_{4}$ are equal to 1, by the multiplication table in [16. Using Theorem 1.1, we can find their corresponding classical intersection numbers, the most complicated case of which has been given in the above example.

The proof of Theorem 1.2 has also shown us how to find $v^{\prime}$ and $w^{\prime}$. Combining Theorem 1.2 and the Peterson-Woodward comparison formula (Proposition 2.1), we can obtain many nice applications, including alternative proofs of both the quantum Pieri rule for all flag varieties of $A$-type given by Ciocan-Fontanine in [10] and the result that any three-pointed genus zero Gromov-Witten invariant on a complex Grassmannian is a classical intersection number on a two-step flag variety of the same type, which is the central theme of 4 for type $A$ case by Buch, Kresch 
and Tamvakis. In order to illustrate this clearly, we will show how to recover the "quantum to classical" principle for complex Grassmannians in the rest.

For the complex Grassmannian $X=G / P=G r(k, n+1)$, we note $H_{2}(X, \mathbb{Z}) \cong$ $Q^{\vee} / Q_{P}^{\vee} \cong \mathbb{Z}$, so that we simply denote $N_{u, v}^{w, d}:=N_{u, v}^{w, \lambda_{P}}$ where $u, v, w \in W^{P}$ and $\lambda_{P}=d \alpha_{k}^{\vee}+Q_{P}^{\vee}$. Write $d=m_{1} k+r_{1}=m_{2}(n-k+1)+r_{2}$ where $1 \leq r_{1} \leq k$ and $1 \leq r_{2} \leq n-k+1$. Then for $\lambda:=m_{1} \sum_{j=1}^{k-1} j \alpha_{j}^{\vee}+\sum_{j=1}^{r_{1}-1} j \alpha_{k-r_{1}+j}^{\vee}+d \alpha_{k}^{\vee}+$ $m_{2} \sum_{j=1}^{n-k+1} j \alpha_{n+1-j}^{\vee}+\sum_{j=1}^{r_{2}-1} j \alpha_{k+r_{2}-j}^{\vee}$, we have $\left\langle\alpha_{i}, \lambda\right\rangle=-1$ if $i \in\left\{k-r_{1}, k+r_{2}\right\}$, or 0 otherwise. Thus it follows directly from the uniqueness of $\lambda_{B}$ that $\lambda_{B}=\lambda$. Furthermore by Proposition 2.1, we have $N_{u, v}^{w, d}=N_{u, v}^{\tilde{w}, \lambda_{B}}$ with

$$
\tilde{w}=w \omega_{P} \omega_{P^{\prime}}=w u_{k-r_{1}}^{(k-1)} u_{k-r_{1}}^{(k-2)} \cdots u_{k-r_{1}}^{\left(k-r_{1}\right)} v_{n+1-k-r_{2}}^{\left(n-r_{2}+1\right)} v_{n+1-k-r_{2}}^{\left(n-r_{2}+2\right)} \cdots v_{n+1-k-r_{2}}^{(n)}
$$

(see e.g. Lemma 3.6 of [30] for the way of obtaining $\omega_{P} \omega_{P^{\prime}}$ ). Here for any $1 \leq i \leq m$, we denote $u_{i}^{(m)}:=s_{m-i+1} \cdots s_{m-1} s_{m}$ and $v_{i}^{(m)}=\left(u_{i}^{(m)}\right)^{-1}=s_{m} s_{m-1} \cdots s_{m-i+1}$; in addition, we denote $u_{0}^{(m)}=v_{0}^{(m)}=\mathrm{id}$.

In particular, if $1 \leq d \leq \min \{k, n+1-k\}$, then we have $d=r_{1}=r_{2}$ and $\Delta_{P^{\prime}}=\Delta_{P} \backslash\left\{\alpha_{k-d}, \alpha_{k+d}\right\}$. Furthermore in this case, we go through the proof of Theorem 1.2 for the above special $\lambda_{B}$, by reducing it to the zero coroot according to the ordering $\left(\left(\alpha_{k}^{\vee}, \alpha_{k-1}^{\vee}, \cdots, \alpha_{k-d+1}^{\vee}\right),\left(\alpha_{k+1}^{\vee}, \alpha_{k+2}^{\vee}, \cdots, \alpha_{k+d-1}^{\vee}\right),\left(\alpha_{k}^{\vee}, \cdots, \alpha_{k-d+2}^{\vee}\right)\right.$, $\left.\left(\alpha_{k+1}^{\vee}, \cdots, \alpha_{k+d-2}^{\vee}\right), \cdots,\left(\alpha_{k}^{\vee}, \alpha_{k-1}^{\vee}\right),\left(\alpha_{k+1}^{\vee}\right), \alpha_{k}^{\vee}\right)$. Correspondingly, we denote

$$
x:=v_{d}^{(k)} u_{d-1}^{k+d-1} v_{d-1}^{(k)} u_{d-2}^{(k+d-2)} \cdots v_{2}^{(k)} u_{1}^{(k+1)} s_{k} .
$$

(Note $\ell(x)=d^{2}$.) As a direct consequence, we have

Corollary 2.12. For any $u, v, w \in W^{P}$ and $d \in \mathbb{Z}$ with $1 \leq d \leq \min \{k, n+1-k\}$, we have $N_{u, v}^{w, d}=N_{u, v x}^{\tilde{w} x, 0}$, provided that $\ell(v x)=\ell(v)-\ell(x)$ and $\ell(\tilde{w} x)=\ell(\tilde{w})+\ell(x)$, and zero otherwise.

Let $\bar{P} \supset B$ denote the parabolic subgroup that corresponds to the subset $\Delta \backslash$ $\left\{\alpha_{k-d}, \alpha_{k+d}\right\}$. That is, $G / \bar{P}=F \ell_{k-d, k+d ; n+1}=\left\{V \leqslant V^{\prime} \leqslant \mathbb{C}^{n+1} \mid \operatorname{dim} V=\right.$ $\left.k-d, \operatorname{dim} V^{\prime}=k+d\right\}$ is a two-step flag variety. We can reprove the next result of Buch, Kresch and Tamvakis.

Proposition 2.13 (Corollary 1 of [4]). For any Schubert classes $\sigma^{u}, \sigma^{v}, \sigma^{w}$ in $H^{*}(G r(k, n+1), \mathbb{Z})$ and any $d \geq 1$, the Gromov-Witten invariant $N_{u, v}^{w, d}$ coincides with the classical intersection number $N_{u x, v x}^{\tilde{w}, 0}$ for $\sigma^{u x} \cup \sigma^{v x}$ in $H^{*}\left(F \ell_{k-d, k+d ; n+1}, \mathbb{Z}\right)$, provided that $d \leq \min \{k, n+1-k\}, \ell(u x)=\ell(u)-\ell(x), \ell(v x)=\ell(v)-\ell(x)$ and $\tilde{w} \in W^{\bar{P}}$, and vanishes otherwise.

To show the above proposition, we need the next two lemmas.

Lemma 2.14. For any Schubert classes $\sigma^{u}, \sigma^{v}, \sigma^{w}$ in $H^{*}(G r(k, n+1), \mathbb{Z})$, the Gromov-Witten invariant $N_{u, v}^{w, d}$ vanishes unless $0 \leq d \leq \min \{k, n+1-k\}$.

Proof. Note $N_{u, v}^{w, d}=N_{u, v}^{\tilde{w}, \lambda_{B}}$. If $k=1$ (resp. $n$ ), then $\left\langle\alpha_{k}, \lambda_{B}\right\rangle=d+m_{2}+1$ (resp. $d+m_{1}+1$ ) is larger than 2 , whenever $d>1=\min \{k, n+1-k\}$. Thus we have $N_{u, v}^{\tilde{w}, \lambda_{B}}=0$ by Theorem 1.1 (1). If $2 \leq k \leq n-1$, then we have $\left\langle\alpha_{k}, \lambda_{B}\right\rangle=$ $m_{1}+m_{2}+2$. By Theorem[1.1 (1) again, we have $N_{u, v}^{\tilde{w}, \lambda_{B}}=0$ unless $m_{1}=m_{2}=0$, in which case we still have $d=r_{1}=r_{2} \leq \min \{k, n+1-k\}$.

Lemma 2.15. For any $v \in W^{P}$, we have $v x \in W^{\bar{P}}$ if $\ell(v x)=\ell(v)-\ell(x)$. 
Proof. Since $\ell(v x)=\ell(v)-\ell(x)$, we have $\ell(v x)=\ell\left(v x s_{k}\right)-1$, so that $v x\left(\alpha_{k}\right) \in R^{+}$. For any $j \in\{1, \cdots, k-d-1, k+d+1, \cdots, n\}$, we have $v x\left(\alpha_{j}\right)=v\left(\alpha_{j}\right) \in R^{+}$. For $j \in\{k-d+1, \cdots, k-1\}$, we have $v x\left(\alpha_{j}\right)=v v_{d}^{(k)} u_{d-1}^{k+d-1} \cdots v_{k-j+1}^{(k)} u_{k-j}^{(2 k-j)} v_{k-j}^{(k)}\left(\alpha_{j}\right)$ $=v v_{d}^{(k)} u_{d-1}^{k+d-1} \cdots v_{k-j+2}^{(k)} u_{k-j+1}^{(2 k-j+1)}\left(\alpha_{k+1}\right)=v\left(\alpha_{k+d}\right) \in R^{+}$. Similarly for $j \in\{k+$ $1, \cdots, k+d-1\}$, we have $v x\left(\alpha_{j}\right)=v\left(\alpha_{j-d}\right) \in R^{+}$. Hence, we have $v x \in W^{\bar{P}}$.

Remark 2.16. The Weyl group $W$ for $G=S L(n+1, \mathbb{C})$ is canonically isomorphic to the permutation group $S_{n+1}$ by mapping each simple reflection $s_{i} \in W$ to the transposition $(i, i+1) \in S_{n+1}$. In "one-line" notation, each permutation $t \in W=$ $S_{n+1}$ is written as $(t(1), \cdots, t(n+1))$. In particular, Grassmannian permutations $t \in W^{P}$ for $G / P=G r(k, n+1)$ are precisely the permutations with (at most) a single descent occurring at $k$ th position (i.e., $t(k)>t(k+1)$ ). Permutations $t \in W^{\bar{P}}$ for $G / \bar{P}=F \ell_{k-d, k+d ; n+1}$ are precisely the permutations with (at most) two descents occurring at $(k-d)$ th and $(k+d)$ th position. With this characterization, $v x \in W^{\bar{P}}$ is the element obtained from $v \in W^{P}$ by sorting the values $\{v(k-d+1), \cdots, v(k+d)\}$ to be in increasing order, which coincides with the descriptions in section 2.2 of [4. ( Indeed, we note that $s_{j}(i)=i$ for any $j \in\{k-d+1, \cdots, k+d-1\}$ and any $i \in\{1, \cdots, k-d, k+d+1, \cdots, n+1\}$. Thus we have $v x(i)=v(i)$ for any such $i$ and consequently the set $\{v(k-d+1), \cdots, v(k+d)\}$ coincides with the set $\{v x(k-d+1), \cdots, v x(k+d)\}$.$) Similarly, we can show that \tilde{w}$ is the permutation $(w(d+1), \cdots, w(k), \tilde{w}(k-d+1), \cdots, \tilde{w}(k+d), w(k+1), \cdots, w(n-d+1))$, in which $(\tilde{w}(k-d+1), \cdots, \tilde{w}(k+d))$ is obtained from $w \in W^{P}$ by sorting the values $\{w(1), \cdots, w(d), w(n-d+2), \cdots, w(n+1)\}$ to be in increasing order.

Proof of Proposition 2.13. It follows from Lemma 2.14 and Corollary 2.12 that $N_{u, v}^{\tilde{w}, d}=N_{u, v x}^{\tilde{w} x, 0}$ if $1 \leq d \leq \min \{k, n+1-k\}, \ell(v x)=\ell(v)-\ell(x)$ and $\ell(\tilde{w} x)=$ $\ell(\tilde{w})+\ell(x)$, or 0 otherwise. When all of these hold, we have $v x \in W^{\bar{P}}$ by Lemma 2.15 and note that $x$ is in the Weyl subgroup generated by $\left\{s_{k-d+1}, \cdots, s_{k+d-1}\right\}$. In particular for any $j \in\{k-d+1, \cdots, k+d-1\}$, we have $\operatorname{sgn}_{j}(v x)=0$. Thus by Theorem 1.1] we have $N_{u, v x}^{\tilde{w} x, 0}=N_{u x^{-1}, v x}^{\tilde{w}, 0}$ provided that $\ell\left(u x^{-1}\right)=\ell(u)-\ell\left(x^{-1}\right)$, and zero otherwise. This assumption implies that $u x \in W^{\bar{P}}$, because of the observation that $x=x^{-1}$. As a consequence, $N_{u x, v x}^{\tilde{w}, 0}=0$ unless $\tilde{w} \in W^{\bar{P}}$, for which the assumption " $\ell(\tilde{w} x)=\ell(\tilde{w})+\ell(x)$ " holds automatically.

\section{Acknowledgements}

The authors thank Ionut Ciocan-Fontanine, Bumsig Kim, Leonardo Constantin Mihalcea and Harry Tamvakis for useful discussions. We also thank the referees for valuable suggestions. The work described in this paper was substantially supported by a grant from the Research Grants Council of the Hong Kong Special Administrative Region, China (Project No. CUHK401908), and partially by the KRF grant 2007-341-C00006.

\section{REFERENCES}

[1] I.N. Bernstein, I.M. Gel'fand and S.I. Gel'fand, Schubert cells and cohomology of the spaces $G / P$, Russian Math. Surveys 28(1973), 1-26.

[2] A. Bertram, Quantum Schubert calculus, Adv. Math. 128 (1997), no. 2, 289-305.

[3] A.S. Buch, Quantum cohomology of Grassmannians, Compositio Math. 137 (2003), no. 2, $227-235$ 
[4] A.S. Buch, A. Kresch and H. Tamvakis, Gromov-Witten invariants on Grassmannians, J. Amer. Math. Soc. 16 (2003), no. 4, 901-915 (electronic).

[5] A.S. Buch, A. Kresch and H. Tamvakis, Quantum Pieri rules for isotropic Grassmannians, Invent. Math. 178 (2009), no. 2, 345-405.

[6] A.S. Buch, L.C. Mihalcea, Quantum K-theory of Grassmannians, Duke Math. J. 156 (2011), no. $3,501-538$.

[7] A.S. Buch, L.C. Mihalcea, Towards a Chevalley formula for the quantum $K$ theory of homogeneous spaces, in preparation.

[8] P.E. Chaput, L. Manivel and N. Perrin, Quantum cohomology of minuscule homogeneous spaces, Transform. Groups 13 (2008), no. 1, 47-89.

[9] P.-E. Chaput, N. Perrin, On the quantum cohomology of adjoint varieties, arXiv: math. AG/0904.4824.

[10] I. Ciocan-Fontanine, On quantum cohomology rings of partial flag varieties, Duke Math. J. 98 (1999), no. 3, 485-524.

[11] I. Coskun, A Littlewood-Richardson rule for two-step flag varieties, Invent. Math. 176 (2009), no. 2, 325-395.

[12] I. Coskun, A Littlewood-Richardson rule for partial flag varieties, preprint.

[13] I. Coskun, The quantum cohomology of flag varieties and the periodicity of the Schubert structure constants, Math. Ann. 346 (2010), no. 2, 419-447.

[14] H. Duan, Multiplicative rule of Schubert class, Invent. Math. 159 (2005), no. 2, 407-436.

[15] H. Duan, N.C. Leung, C. Li and X. Zhao, in preparation.

[16] S. Fomin, S. Gelfand and A. Postnikov, Quantum Schubert polynomials, J. Amer. Math. Soc. 10 (1997), no. 3, 565-596.

[17] W. Fulton, On the quantum cohomology of homogeneous varieties, The legacy of Niels Henrik Abel, 729-736, Springer, Berlin, 2004.

[18] W. Fulton, R. Pandharipande, Notes on stable maps and quantum cohomology, Proc. Sympos. Pure Math. 62, Part 2, Amer. Math. Soc., Providence, RI, 1997.

[19] W. Fulton, C. Woodward, On the quantum product of Schubert classes, J. Algebraic Geom. 13 (2004), no. 4, 641-661.

[20] P. Griffiths, J. Harris, Principles of algebraic geometry, John Wiley \& Sons, Inc., New York, 1994.

[21] J.E. Humphreys, Introduction to Lie algebras and representation theory, Graduate Texts in Mathematics 9, Springer-Verlag, New York-Berlin, 1980.

[22] J.E. Humphreys, Linear algebraic groups, Graduate Texts in Mathematics 21, SpringerVerlag, New York-Berlin, 1975.

[23] J.E. Humphreys, Reflection groups and Coxeter groups, Cambridge University Press, Cambridge, UK, 1990.

[24] B. Kim, Quantum cohomology of flag manifolds $G / B$ and quantum Toda lattices, Ann. of Math. (2) 149 (1999), no. 1, 129-148.

[25] B. Kostant, S. Kumar, The nil Hecke ring and the cohomology of G/P for a Kac-Moody group G, Adv. in Math. 62 (1986), 187-237.

[26] A. Kresch, H. Tamvakis, Quantum cohomology of the Lagrangian Grassmannian, J. Algebraic Geom. 12 (2003), no. 4, 777-810.

[27] A. Kresch, H. Tamvakis, Quantum cohomology of orthogonal Grassmannians, Compos. Math. 140 (2004), no. 2, 482-500.

[28] T. Lam, M. Shimozono, Quantum cohomology of $G / P$ and homology of affine Grassmannian, Acta Math. 204 (2010), no. 1, 49-90.

[29] N.C. Leung, C. Li, Gromov-Witten invariants for $G / B$ and Pontryagin product for $\Omega K$, to appear in Trans. Amer. Math. Soc., arXiv: math. AG/0810.4859.

[30] N.C. Leung, C. Li, Functorial relationships between $Q H^{*}(G / B)$ and $Q H^{*}(G / P)$, J. Differential Geom. 86 (2010), no. 2, 303-354.

[31] N.C. Leung, C. Li, Quantum Pieri rules for tautological subbundles, preprint.

[32] C. Li, L.C. Mihalcea, Spaces of lines in $G / B$, in preparation.

[33] D. Peterson, Quantum cohomology of $G / P$, Lecture notes at MIT, 1997 (notes by J. Lu and K. Rietsch).

[34] A. Postnikov, Symmetries of Gromov-Witten invariants, Advances in algebraic geometry motivated by physics (Lowell, MA, 2000), 251-258, Contemp. Math., 276, Amer. Math. Soc., Providence, RI, 2001. 
[35] B. Siebert, G. Tian, On quantum cohomology rings of Fano manifolds and a formula of Vafa and Intriligator, Asian J. Math. 1 (1997), no. 4, 679-695.

[36] C.T. Woodward, On D. Peterson's comparison formula for Gromov-Witten invariants of G/P, Proc. Amer. Math. Soc. 133 (2005), no. 6, 1601-1609.

The Institute of Mathematical Sciences and Department of Mathematics, The ChiNese University of Hong Kong, Shatin, Hong Kong

E-mail address: leung@math.cuhk.edu.hk

School of Mathematics, Korea Institute for Advanced Study, 87 Hoegiro, DongdaemunGu, Seoul, 130-722, Korea

E-mail address: czli@kias.re.kr 